\title{
Research on Path Planning Method of Coal Mine Robot to Avoid Obstacle in Gas Distribution Area
}

\author{
Ruiqing $\mathrm{Mao}^{1,2}$ and Xiliang $\mathrm{Ma}^{1,2}$ \\ ${ }^{1}$ Xuzhou Institute of Technology, Xuzhou, Jiangsu 221111, China \\ ${ }^{2}$ School of Mechanical and Electrical Engineering, China University of Mining and Technology, Xuzhou, Jiangsu 221116, China
}

Correspondence should be addressed to Xiliang Ma; xlma818@sina.com

Received 24 February 2016; Accepted 10 July 2016

Academic Editor: Gordon R. Pennock

Copyright (c) 2016 R. Mao and X. Ma. This is an open access article distributed under the Creative Commons Attribution License, which permits unrestricted use, distribution, and reproduction in any medium, provided the original work is properly cited.

As the explosion-proof safety level of a coal mine robot has not yet reached the level of intrinsic safety "ia" and it cannot work in a dangerous gas distribution area, therefore, path planning methods for coal mine robot to avoid the dangerous area of gas are necessary. In this paper, to avoid a secondary explosion when the coal mine robot passes through gas hazard zones, a path planning method is proposed with consideration of gas concentration distributions. First, with consideration of gas distribution area and obstacles, MAKLINK method is adopted to describe the working environment network diagram of the coal mine robot. Second, the initial working paths for the coal mine robot are obtained based on Dijkstra algorithm, and then the global optimal working path for the coal mine robot is obtained based on ant colony algorithm. Lastly, experiments are conducted in a roadway after an accident, and results by different path planning methods are compared, which verified the effectiveness of the proposed path planning method.

\section{Introduction}

Environment exploration (such as detection of methane concentration) is often necessary before coal mining, and a coal mine robot is needed. Similarly, rescue must be carried out after a coal mine accident, and the working environment in the roadway is always very complex; therefore, coal mine robot is also a good choice. In the process of environment detection or rescue, a secondary explosion must be avoided.

In China, the coal mine safe electrical apparatus is Class I, which is strict with the use of mining equipment. Therefore, with regard to coal mine robot, the explosion-proof grade needs to reach the level of intrinsic safety "ia." However, at present, very few apparatuses can reach the level of "ia" [1]. In order to promote the utility of coal mine robot and avoid the danger during the working process, studies on path planning for coal mine robot are of great significance. On the other hand, the existing studies demonstrated that gas in roadways has the regularities of regional distribution and migration $[2,3]$. And the authors of this paper have studied the methane concentration prediction method in the previous studies, as seen in [4].

Path planning, which can find out the optimal working path without obstacles, is very important for coal mine robot. Generally, path planning is a nondeterministic polynomial problem with such constraints from much information of environment and obstacles [5]. Artificial Potential Field Method [6], Grid Method [7], and Visibility Graph Method [8] are the common path planning methods. These methods have their own shortages. For example, Artificial Potential Field Method is prone to fall into local optimal point; in complex environment, a surge of storage capacity may arise if Grid Method is adopted; Visibility Graph Method has such shortages as high searching complexity and low efficiency. Pan et al. [9] applied an improved ant colony algorithm to the path planning for mobile robot with peaks on the path considered. Zhang et al. [10] proposed a global path planning method for coal mine rescue robot based on ant colony algorithm. Li et al. [11] studied the global path planning method for mobile robot based on Particle Swarm 
Optimization. The complex working environment in roadway after coal mine accident brings about great challenges to the path planning for coal mine robot. And there are few researches on path planning method for coal mine robot with gas concentration distributions considered.

In the paper, to avoid a secondary explosion when the coal mine robot passes through gas hazard zones, path planning method is studied. First, MAKLINK method is adopted to describe the working environment network diagram of the coal mine robot with consideration of gas distribution area and obstacles. Second, with the help of Dijkstra algorithm, the initial working paths for coal mine robot are obtained, and then ant colony algorithm is adopted to optimize the initial working paths, and the global optimal working paths for coal mine robot are obtained. Lastly, experiments are conducted in a roadway after an accident, and results by different path planning methods are compared.

\section{Path Planning for Coal Mine Robot}

In this paper, with consideration of gas distribution area and obstacles, MAKLINK method is adopted to describe the working environment network graph of coal mine robot. Suppose that the roadway bottom is parallel to $X Y$ plane of the space rectangular coordinate system $O X Y Z$ and the height of gas concentration distributions and obstacles is parallel to $z$ axis. The lines linking the nodes, which do not intersect with the obstacles and gas concentration distribution areas, are defined as MAKLINK lines. The convex polygon areas, which are divided by the MAKLINK lines, make up the feasible working space of coal mine robot. Based on the feasible working space, the midpoints of the MAKLINK lines are defined as the possible path nodes $v_{1}, v_{2}, \ldots, v_{n}$. The feasible paths of coal mine robot are formed by the lines that link the starting point, the possible path nodes [12], and the target point.

Based on the GIS system before the mine accident and the modeling of roadway environment after the mine accident, the optimal working path for coal mine robot is obtained by Dijkstra-ant colony algorithm. The optimal working path planning method for coal mine robot is shown in Figure 1.

2.1. Initial Path Planning for Coal Mine Robot. The initial working paths for coal mine robot are obtained based on Dijkstra algorithm. The working environment network graph obtained by MAKLINK method is defined as $G=(V, E)$, where $V$ is the set of all the path nodes which include the starting point and the target point. $E$ is the set of edges which link the path nodes.

The adjacency matrix of the network graph $G=(V, E)$ is defined as $X$. The value of the element at $i$ th row and $j$ th column can be expressed as follows [13]:

$$
X_{i, j}= \begin{cases}H\left(v_{i}, v_{j}\right) & v_{i} \text { and } v_{j} \text { neighboring } \\ \infty & v_{i} \text { and } v_{j} \text { not neighboring }\end{cases}
$$

where $H\left(v_{i}, v_{j}\right)$ is the distance between $v_{i}$ and $v_{j}$.

The set of path nodes of coal mine robot is divided into two subsets $S$ and $U$, and $V=\{S, U\}$, where $S$ is the set of path nodes that have been admitted to be on the shortest path and $U$ is the set of the path nodes that have not.

Based on Dijkstra algorithm, the steps to obtain the initial working paths for coal mine robot are as follows:

(1) Put the starting point St into $S$ and the rest of the path nodes into $U$. The adjacency matrix $X$ is used to initialize the shortest paths $W$ between St and each point in $U$.

(2) Select the minimum value $W_{i}$ in $W$, and put the corresponding point $v_{i}$ into $S$.

(3) Use point $v_{i}$ as the intermediate point, and update the distances $W^{\prime}$ between $S t$ and each point in $U$. If the direct distance between St and $v_{j}$ in $U$ is larger than the sum of the distance between St and $v_{i}$ and that between $v_{i}$ and $v_{j}$, the distance between St and $v_{j}$ is updated to be $W_{j}^{\prime}=W_{i}+H\left(v_{i}, v_{j}\right)$.

(4) Repeat step (2) and step (3), until the set $U$ is empty. The shortest paths between St and each path node are obtained.

2.2. Optimal Path Planning for Coal Mine Robot. As it is known that a safe working path for coal mine robot can be determined through Dijkstra algorithm, due to the inherent shortcomings of Dijkstra algorithm, it is difficult to guarantee that the obtained path is the optimal one. Therefore, to get the optimal working path for coal mine robot, the initial working paths obtained by Dijkstra algorithm are further optimized by ant colony algorithm.

A working path from St to Ds for coal mine robot obtained by Dijkstra algorithm can be expressed as follows:

$$
\text { St } \longrightarrow n_{1} \longrightarrow n_{2} \longrightarrow \cdots \longrightarrow n_{l} \longrightarrow \text { Ds, }
$$

where $n_{i}(i=1,2, \ldots, l)$ is the sequential path nodes.

The initial length of the paths and the distances between the path nodes and the nodes of obstacles or gas concentration distributions should be considered simultaneously when ant colony algorithm is used. The objective function of the optimal working path for coal mine robot is defined as follows [14]:

$$
F_{\text {goal }}=H^{k}+\frac{1}{\min \left(d_{\mathrm{ob}}^{k}\right)}
$$

where $H^{k}$ is the length of the path that is passed by the $k$ th ant; $\min \left(d_{\mathrm{ob}}^{k}\right)$ is the minimum value of the distance between the path nodes and the nodes of obstacles or gas concentration distributions passed by the $k$ th ant.

The edges on the initial path in MAKLINK graph are defined as $H_{i}(i=1,2, \ldots, l)$. The endpoints of $H_{i}$ are $v_{i, 0}$ and $v_{i, 1}$. Other path nodes can be expressed as follows:

$$
v_{i}=v_{i, 0}+\left(v_{i, 1}-v_{i, 0}\right) \times k_{i},
$$

where $k_{i}$ is a scale factor and $k_{i} \in[0,1]$.

For a given set of parameters $k_{i}(i=1,2, \ldots, l)$, a new path can be obtained by the determined new path nodes on each 


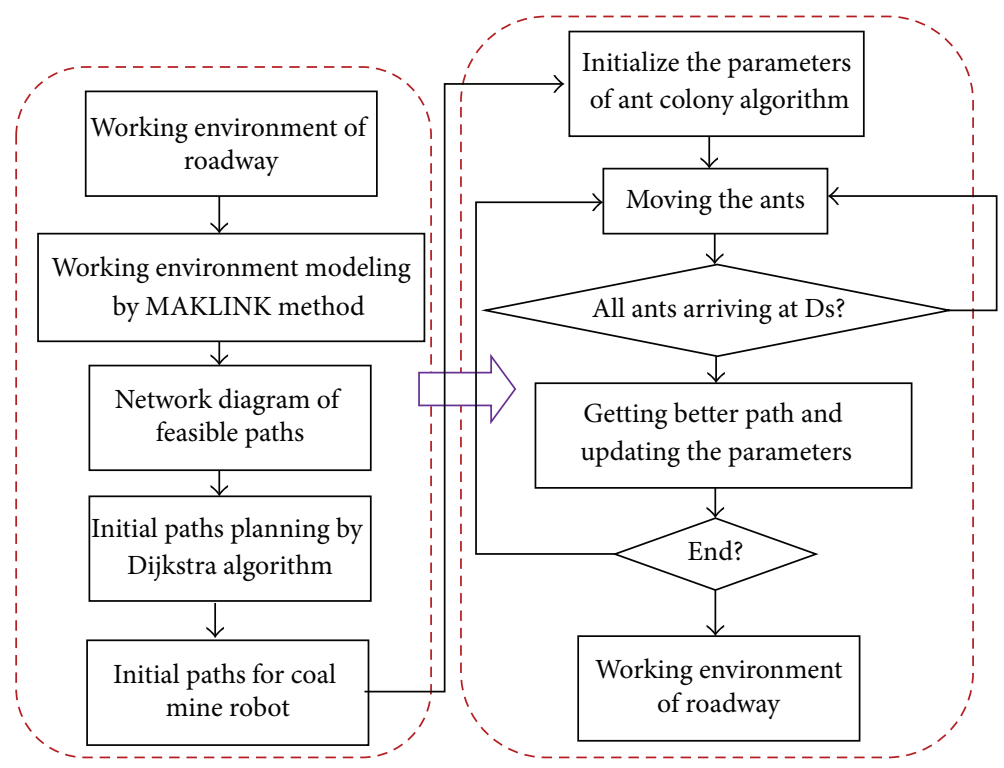

FIGURE 1: Dijkstra-ant colony algorithm for coal mine robot.

$H_{i}$. The path with the minimum function value is the optimal one for coal mine robot.

The initial path $H_{i}$ is split by a fixed-length $\Delta H$. The numbers of the splits of each initial path $H_{i}$ can be expressed as follows:

$$
n\left(L_{i}\right)= \begin{cases}\left\lfloor\frac{H_{i}}{\Delta H}\right\rfloor & \left\lfloor\frac{H_{i}}{\Delta H}\right\rfloor \text { is an even number } \\ \left\lfloor\frac{H_{i}}{\Delta H}\right\rfloor+1 & \left\lfloor\frac{H_{i}}{\Delta H}\right\rfloor \text { is an odd number. }\end{cases}
$$

There are $n\left(H_{i+1}\right)+1$ selective paths from each node on edge $H_{i}$ to the adjacent edge $H_{i+1}$. Supposing that the initial ant quantity of ant colony algorithm is $m$, the path from the starting point St to the target point Ds can be expressed as follows:

$$
\text { St } \longrightarrow n_{1 j} \longrightarrow n_{2 j} \longrightarrow \cdots \longrightarrow n_{l j} \longrightarrow \text { Ds, }
$$

where $n_{l j}$ represents the $j$ th equal division point of the path node on the edge $H_{l}$.

During ant colony algorithm solving process, to avoid falling into a local optimum, a randomly selected parameter $q(q \in[0,1])$ is defined as the selective probability when the $k$ th ant moves from $v_{i}$ to $v_{j}$. The selective probability is compared with a scheduled selectivity constant $q_{0}\left(q_{0} \in\right.$ $[0,1])$, and the final selective probability is determined by the compared result and can be expressed as follows:

$$
P_{i j}^{k}= \begin{cases}\max \left(\tau_{i, j}{ }^{\varepsilon} \eta_{i, j}{ }^{\delta}\right) & q<q_{0} \\ \frac{\tau_{i, j}{ }^{\varepsilon} \eta_{i, j}{ }^{\delta}}{\sum_{s \text { allowed }_{k}} \tau_{i, s}{ }^{\varepsilon} \eta_{i, s}{ }^{\delta}} & q \geq q_{0},\end{cases}
$$

where $\tau_{i, j}$ is the pheromone concentration between $v_{i}$ and $v_{j} ; \eta_{i, j}$ is the inspired value between $v_{i}$ and $v_{j}, \eta_{i, j}=1 / d_{i, j}$; $d_{i, j}$ is the distance between $v_{i}$ and $v_{j}$; allowed $_{k}$ is the next elective nodes set of the $k$ th ant; $\varepsilon$ and $\delta$ are the relative importance degree of the pheromone concentration and the inspired value, respectively.

After the selective probability of the $k$ th ant which moves from $v_{i}$ to $v_{j}$ is obtained, a roulette method is used to determine the right node $v_{j}$ to which the $k$ th ant moves. As the $k$ th ant reaches $v_{j}$, pheromone concentration of the node should be updated as follows:

$$
\tau_{i, j}=(1-\rho) \tau_{i, j}^{\prime}+\rho \tau_{0},
$$

where $\tau_{i, j}$ is the pheromone concentration between $v_{i}$ and $v_{j}, \tau_{i, j}^{\prime}$ is the pheromone concentration between $v_{i}$ and $v_{j}$ before update, $\tau_{0}$ is the initial pheromone concentration, $\rho$ is the volatile coefficient of pheromone concentration, and $\rho \subset[0,1]$.

After all the $m$ ants arrive at the target point Ds, the path with the minimum objective function value is selected, and the pheromone concentration of every node on this path is updated as follows:

$$
\tau_{i, j}=(1-\rho) \tau_{i, j}^{\prime}+\rho \Delta \tau_{i, j}
$$

where $\Delta \tau_{i, j}$ is the increment of the pheromone concentration between $v_{i}$ and $v_{j}$ on this cycle path, $\Delta \tau_{i, j}=1 / \min \left(F_{\text {goal }}\right)$, and $\min \left(F_{\text {goal }}\right)$ is the minimum value of the objective function for the paths that all the $m$ ants have passed.

By using ant colony algorithm, the steps of the working path optimization for the rescue robot can be described as follows [15]:

(1) Get the initial path by Dijkstra algorithm, and initialize parameters of ant colony algorithm.

(2) Calculate the selective probability of each ant, and employ a roulette method to determine which node the ant will move to, and move the ant. 
(3) Update the pheromone concentration.

(4) Repeat step (2) and step (3) until all the ants arrive at Ds.

(5) Compute the objective functions $F_{\text {igoal }}(i=1,2, \ldots$, $m$ ) of each ant, and compare $F_{\text {igoal }}$ with the current minimum value $F_{\text {goal min }}$; if $F_{\text {igoal }}<F_{\text {goal min }}$, let $F_{\text {goal min }}=F_{\text {igoal }}$. Then, the pheromone concentration of the nodes that correspond to $F_{\text {igoal }}$ is updated according to (9).

(6) If the loop ends, export the optimal path; otherwise, go to step (2).

The proposed path planning method is suitable for the coal mine robot that works in the roadway of longwall mine.

\section{Experiments and Results}

3.1. Experimental Conditions. The experiments are carried out in the roadway of Huainan Panyi Mine, whose length is 50 meters and width is 3 meters. In the roadway, gas is distributed regionally and the gas concentration is not over the standard $(\leq 5 \%)$. To ensure the safety of the experiments, the area is assumed to be a gas hazard area if the area gas concentration is greater than $2 \%$. Additionally, the length and width of the coal mine robot are $600 \mathrm{~mm}$ and $450 \mathrm{~mm}$, respectively. The picture of the coal mine robot is shown in Figure 2. The coal mine robot is equipped with multiparameter gas detector, which can be used to detect methane, carbon dioxide, carbon monoxide, oxygen, wind speed and direction, temperature, humidity, and so on. It can also be used to predict the gas concentration ahead. Laser radar and infrared range sensors are also equipped on the coal mine robot. A sensing space corresponding to the real environment is built through laser perception. The distance between the robot and the obstacle is detected by the infrared distance sensor. The designed walking speed of the coal mine robot is $1 \mathrm{~m} / \mathrm{s}$ [16]. During the walking process of the coal mine robot, the landform would be evaluated, and if the landform could meet the requirements of walking, the coal mine robot would be walking at the speed of $1 \mathrm{~m} / \mathrm{s}$, which will reduce quickly to $0.2 \mathrm{~m} / \mathrm{s}$ when the obstacle or the gas distribution area is about 3 meters far away. Then, the coal mine robot will go around the obstacle or the gas distribution area at a low speed. In the gas detection process, gas densities are recorded once every $0.5 \mathrm{~m}$ in length and width directions, and then gas distribution map is generated according to the gas concentration. Path planning for the coal mine robot is performed under two different circumstances. In one circumstance, it is assumed that the roadway is a flat one; the robot can gain access through the tunnel except the side wall and the dangerous area of the gas. In the other circumstance, it is assumed that other obstacles exist in the roadway, such as stones, mound, and gas danger area.

3.2. Working Environment Modeling for Rescue Robot. The data collected by the coal mine robot is processed, and the gas concentration distributions are shown in Figure 3. Not all regions in Figure 3 meet the conditions of a gas explosion,

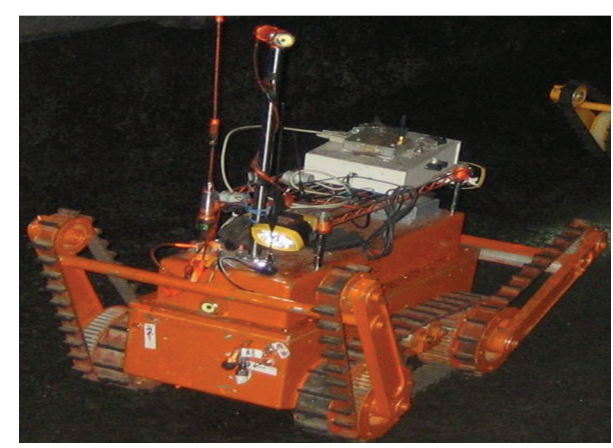

FIgURE 2: The coal mine robot.

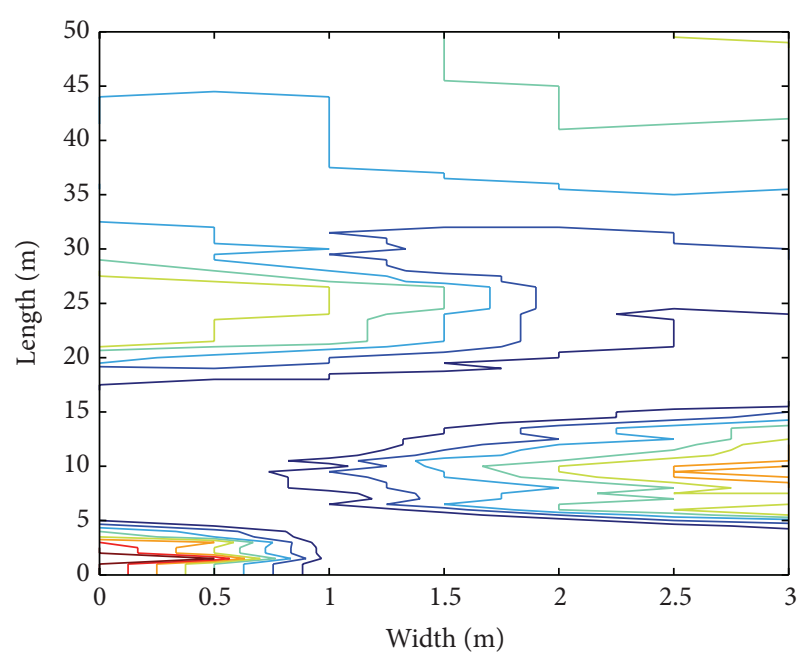

FIGURE 3: Gas concentration distributions.

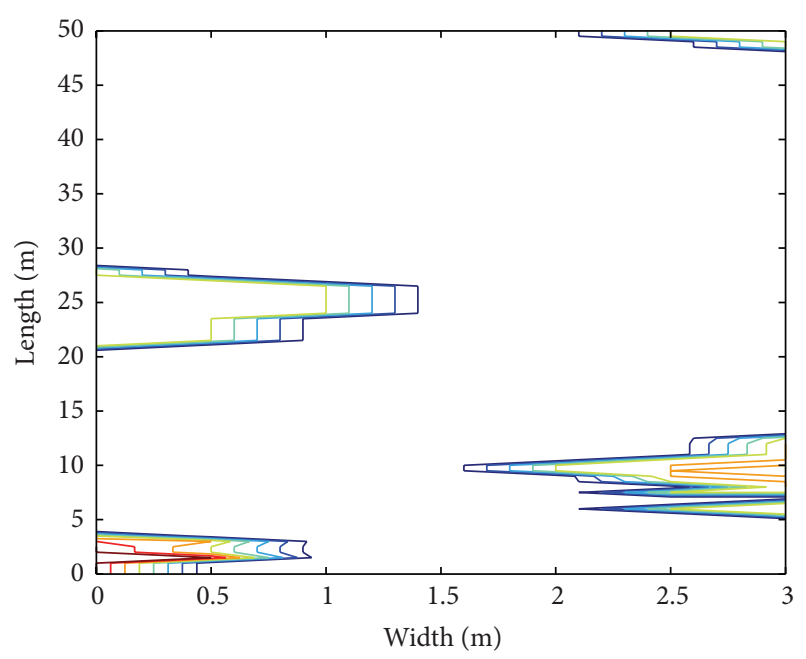

FIGURE 4: Regional distributions of gas prone to explosion.

and, in order to increase the working space of the coal mine robot and avoid a secondary explosion when passing through gas hazard zones, the gas concentration distributions are disposed, which is shown in Figure 4. In Figures 3 and 4, the maximum concentration of the gas exists in the innermost of the closed curve. Although the high concentration of gas 


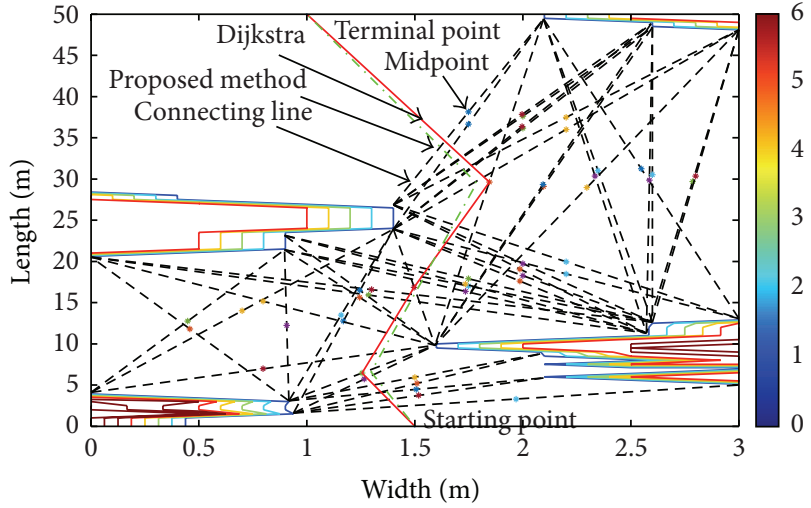

(a) Result of the path planning

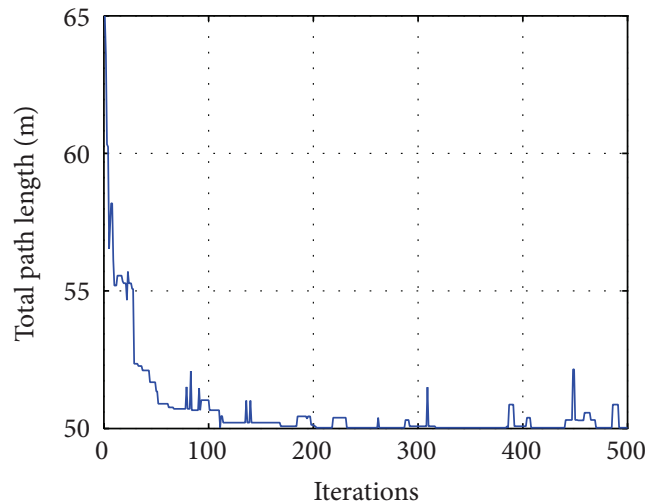

(b) Variation of the total path length

FIGURE 5: Result of the path planning with only consideration of gas concentration distributions. * denotes the midpoint of the MAKLINK lines.

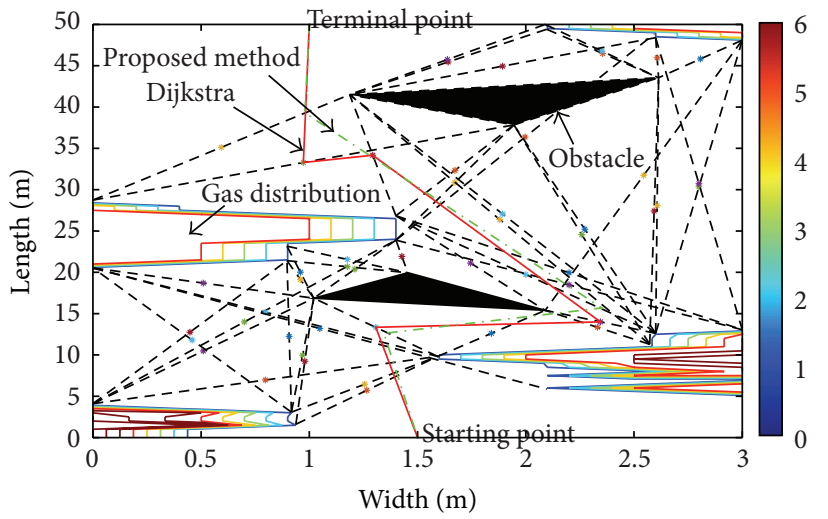

(a) Result of the path planning

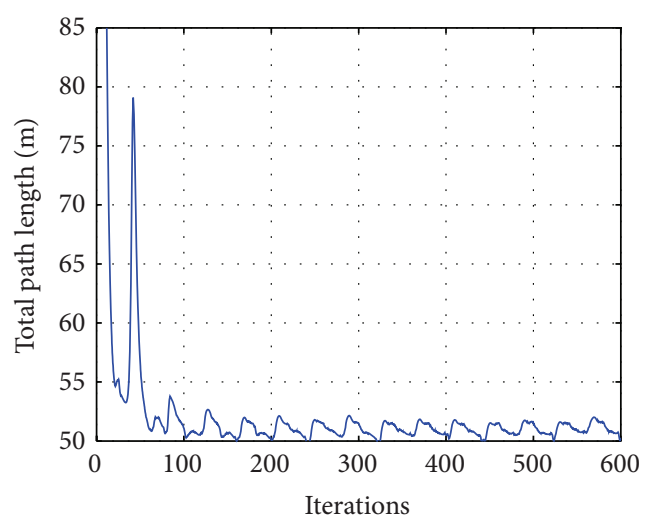

(b) Variation of the total path length

FIGURE 6: Result of the path planning with consideration of gas concentration distributions and obstacles. * denotes the midpoint of the MAKLINK lines.

may not cause an explosion, the coal mine robot cannot pass through.

The proposed method is applied to the working path planning for the coal mine robot with only the gas concentration area distributions. The coordinate of the starting point and the target point for the rescue robot is $(1.5 \mathrm{~m}, 0)$ and $(1 \mathrm{~m}, 50 \mathrm{~m})$, respectively. Dijkstra algorithm and Dijkstra-ant colony optimization algorithm are used to solve the optimal path planning of coal mine robot. The parameters of ant colony algorithm are listed as follows: $\varepsilon=1, \delta=1.2, q_{0}=0.8$, $\rho=0.15, \tau_{0}=0.0004$, and $m=30$, and the maximum iteration is 500 . The final results of the path planning are shown in Figure 5.

As observed from Figure 5, both the planning working paths by Dijkstra algorithm and the proposed method can avoid the danger gas zones. The curve of the total path length is converged at about 100 times. The total path length by Dijkstra algorithm is $63.39 \mathrm{~m}$, while the one by the proposed method is $60.71 \mathrm{~m}$. Therefore, it can be concluded that the proposed method possesses better performance with only consideration of the gas concentration distributions.

The working environment in the roadway is always very complex after a coal mine accident. The proposed method is also applied to the working path planning for the coal mine robot with consideration of the gas concentration distributions and obstacles simultaneously. The final results of the path planning are shown in Figure 6.

As observed from Figure 6, both the planning working paths by Dijkstra algorithm and the proposed method can avoid the danger gas zones with consideration of the gas concentration distributions and obstacles simultaneously. The curve of the total path length is also converged at about 100 times. The total path length by Dijkstra algorithm is $85.61 \mathrm{~m}$, while the one by the proposed method is $73.95 \mathrm{~m}$. Therefore, it can be concluded that the proposed method possesses better performance.

From Figures 5 and 6, it can be seen that the planning paths through the proposed method are shorter, which is of 
great significance to the battery powered coal mine robot. Therefore, the proposed path planning method possesses better performance.

\section{Conclusions}

To avoid a secondary explosion when the coal mine robot passes through gas hazard zones, a path planning method is proposed with consideration of gas concentration distributions and obstacles simultaneously. First, MAKLINK method is adopted to describe the working environment network diagram of the coal mine robot. Second, the initial working paths for the coal mine robot are obtained based on Dijkstra algorithm, and then the global optimal working path for the coal mine robot is obtained based on ant colony algorithm. To verify the effectiveness of the proposed method, lastly, experiments are conducted in a roadway after an accident, and results by different path planning methods are compared. The experiments are divided into two cases; in the first case, it is assumed that the roadway is a flat roadway, and the robot can gain access through the tunnel except the side wall and the dangerous area of the gas; in the other case, obstacles exist in the roadway, such as stones, mound, and gas danger area.

With only consideration of gas concentration distributions, the total path length by Dijkstra algorithm is $63.39 \mathrm{~m}$, while the one by the proposed method is $60.71 \mathrm{~m}$. With consideration of gas concentration distributions and obstacles, the total path length by Dijkstra algorithm is $63.39 \mathrm{~m}$, while the one by the proposed method is $60.71 \mathrm{~m}$. From the results of the path planning with only consideration of gas concentration distributions and the path planning with consideration of gas concentration distributions and obstacles, it can be seen that the planning paths through the proposed method are shorter, which is of great significance to the battery powered coal mine robot. Therefore, the proposed path planning method possesses better performance. The experimental results verified the effectiveness of the proposed path planning method.

\section{Competing Interests}

The authors declare that they have no competing interests.

\section{Acknowledgments}

This work is supported by a grant of the National 863 Program of China (no. 2012AA041504).

\section{References}

[1] D. M. Yang, L. Wang, J. Chen et al., "The basic safety requirements of the coal mine rescue robot," Coal Mine Safety, vol. 42, no. 12, pp. 104-107, 2009.

[2] R.-J. Si, R.-Z. Li, L. Wang, and Z.-K. Wu, "Experiment study on the propagation laws of gas and coal dust explosion in coal mine," Journal of Coal Science \& Engineering, vol. 15, no. 3, pp. 262-266, 2009.
[3] A. P. Sasmito, E. Birgersson, H. C. Ly, and A. S. Mujumdar, "Some approaches to improve ventilation system in underground coal mines environment-a computational fluid dynamic study," Tunnelling and Underground Space Technology, vol. 34, no. 1, pp. 82-95, 2013.

[4] X. Ma and H. Zhu, "Gas concentration prediction based on the measured data of a coal mine rescue robot," Journal of Robotics, vol. 2016, Article ID 6858970, 10 pages, 2016.

[5] B. Huang and R. Kadall, Dynamic Modeling, Predictive Control and Performance Monitoring: A Data-Driven Subs-Pace Approach, Springer, London, UK, 2008.

[6] S. S. Ge and Y. J. Cui, "New potential functions for mobile robot path planning," IEEE Transactions on Robotics and Automation, vol. 16, no. 5, pp. 615-620, 2000.

[7] L. Zhu, J.-Z. Fan, J. Zhao, X.-G. Wu, and G. Liu, "Global path planning and local obstacle avoidance of searching robot in mine disasters based on grid method," Journal of Central South University (Science and Technology), vol. 42, no. 11, pp. 34213428, 2011.

[8] H.-Q. Yang, X.-G. Xiao, and D. Yao, "A V-graph based global path planning algorithm for mobile robot," Journal of Shenyang University of Technology, vol. 31, no. 2, pp. 225-228, 2009.

[9] J. Pan, X.-S. Wang, and Y.-H. Cheng, "Improved ant colony algorithm for mobile robot path planning," Journal of China University of Mining and Technology, vol. 41, no. 1, pp. 108-113, 2012.

[10] X. Y. Zhang, X. Y. Zhou, and J. Wei, "Global path planning for coal mine rescue robot," Journal of Xian University of Science and Technology, vol. 28, no. 2, pp. 323-325, 2008.

[11] Q. Li, Y. M. Xu, D. Z. Zhang, and Y. X. Yin, "Global path planning method for mobile robot based on the particle swarm algorithm," Journal of University of Science and Technology Beijing, no. 3, pp. 397-402, 2010.

[12] F. Wang and H.-Y. Wang, "A re-routing path planning method based on Maklink graph and GA algorithm," Journal of Transportation Systems Engineering and Information Technology, vol. 14, no. 5, pp. 154-160, 2014.

[13] G.-Z. Tan, H. He, and A. Sloman, "Global optimal path planning for mobile robot based on improved Dijkstra algorithm and ant system algorithm," Journal of Central South University of Technology, vol. 13, no. 1, pp. 80-86, 2006.

[14] C. Jin, S. G. Wang, and Y. Y. Yin, "Path planning for multi-robot rescue system under coal mine," Machine Tool and Hydraulics, vol. 42, no. 15, pp. 10-14, 2014.

[15] Y. Cen, C. Song, N. Xie, and L. Wang, "Path planning method for mobile robot based on ant colony optimization algorithm," in Proceedings of the 3rd IEEE Conference on Industrial Electronics and Applications (ICIEA '08), pp. 298-301, Singapore, June 2008.

[16] W. Wang, Z. Du, and L. Sun, "Obstacle performance analysis of mine research robot based on terramechanics," in Proceedings of the IEEE International Conference on Mechatronics and Automation (ICMA '07), pp. 1382-1387, IEEE, Harbin, China, August 2007. 


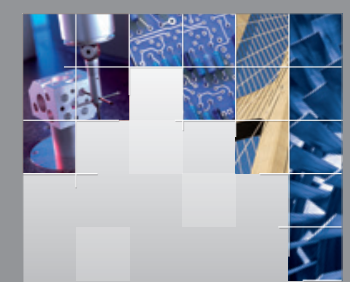

\section{Enfincering}
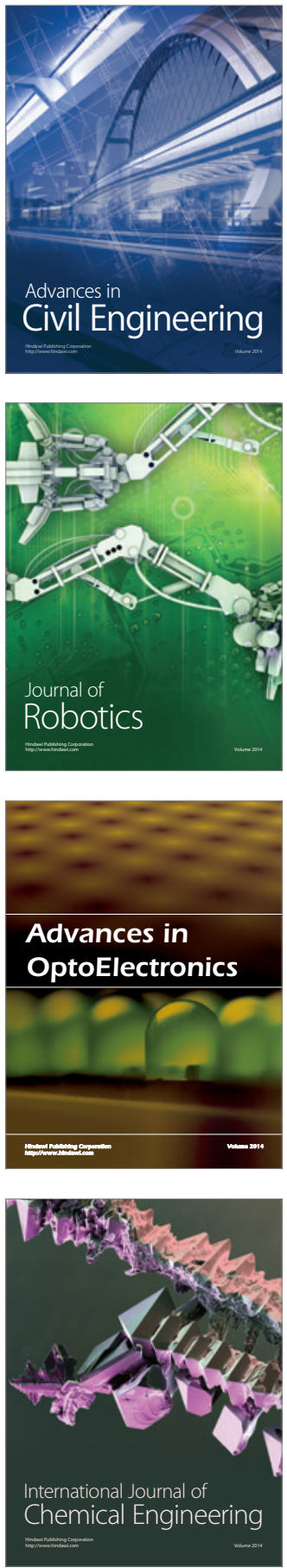

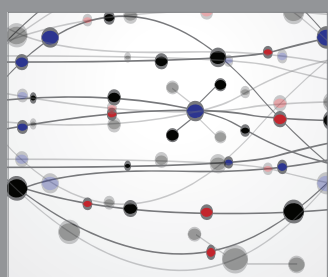

The Scientific World Journal

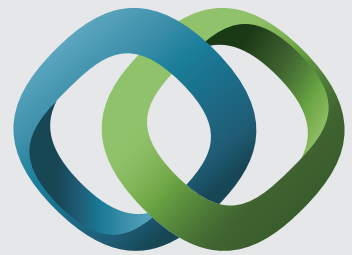

\section{Hindawi}

Submit your manuscripts at

http://www.hindawi.com
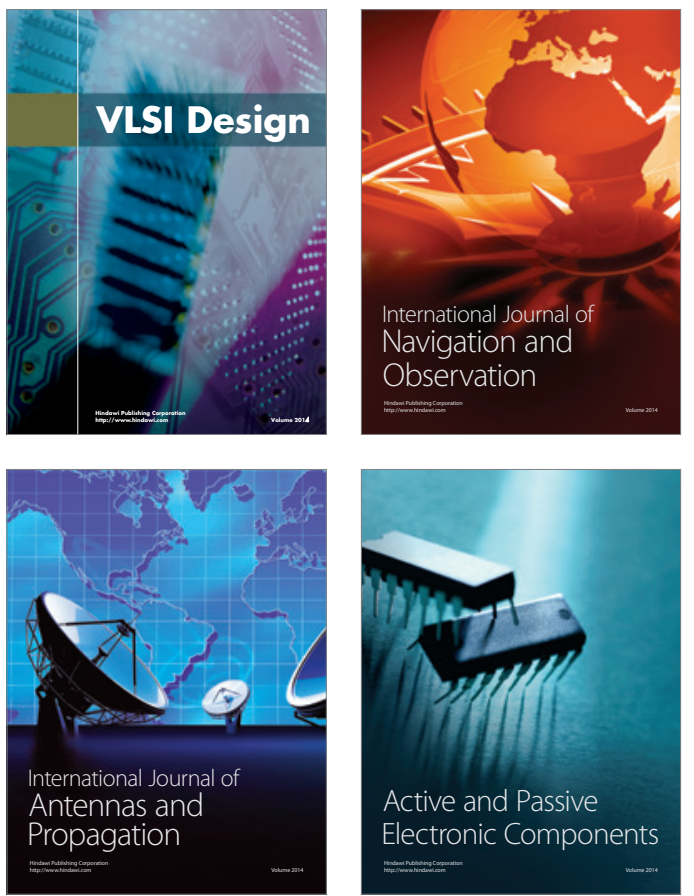
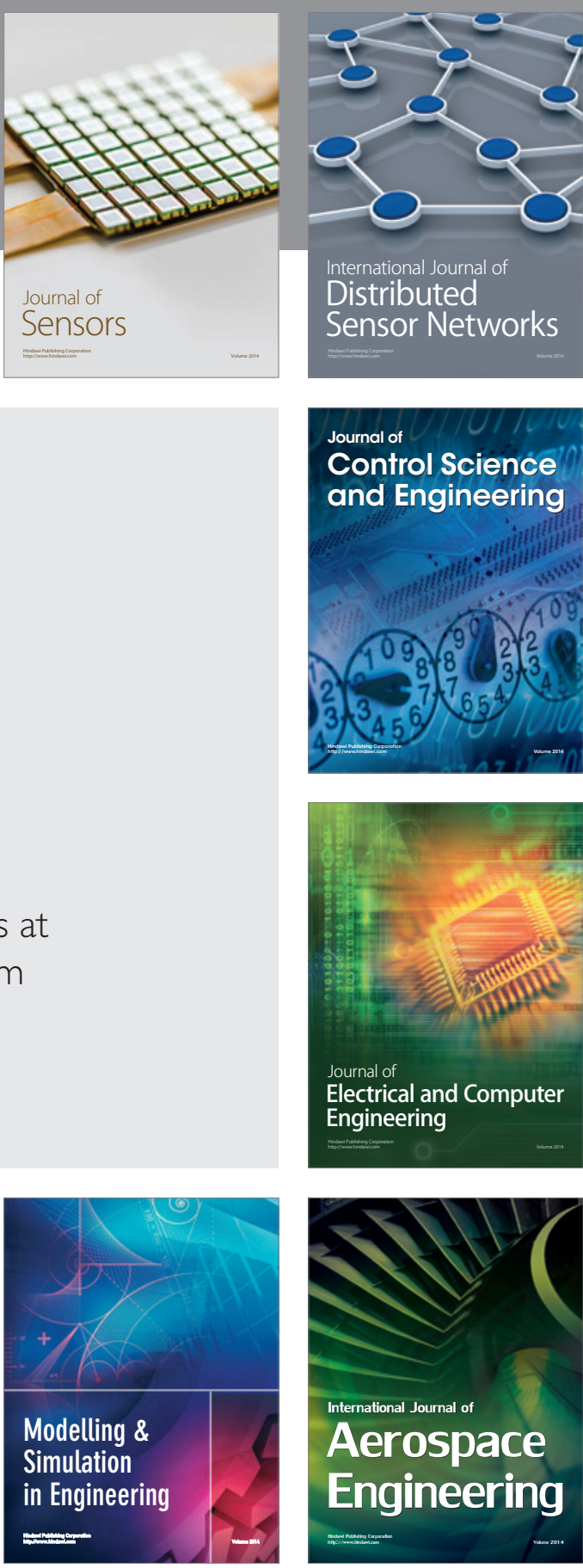

International Journal of

Distributed

Sensor Networks

Journal of

Control Science

and Engineering
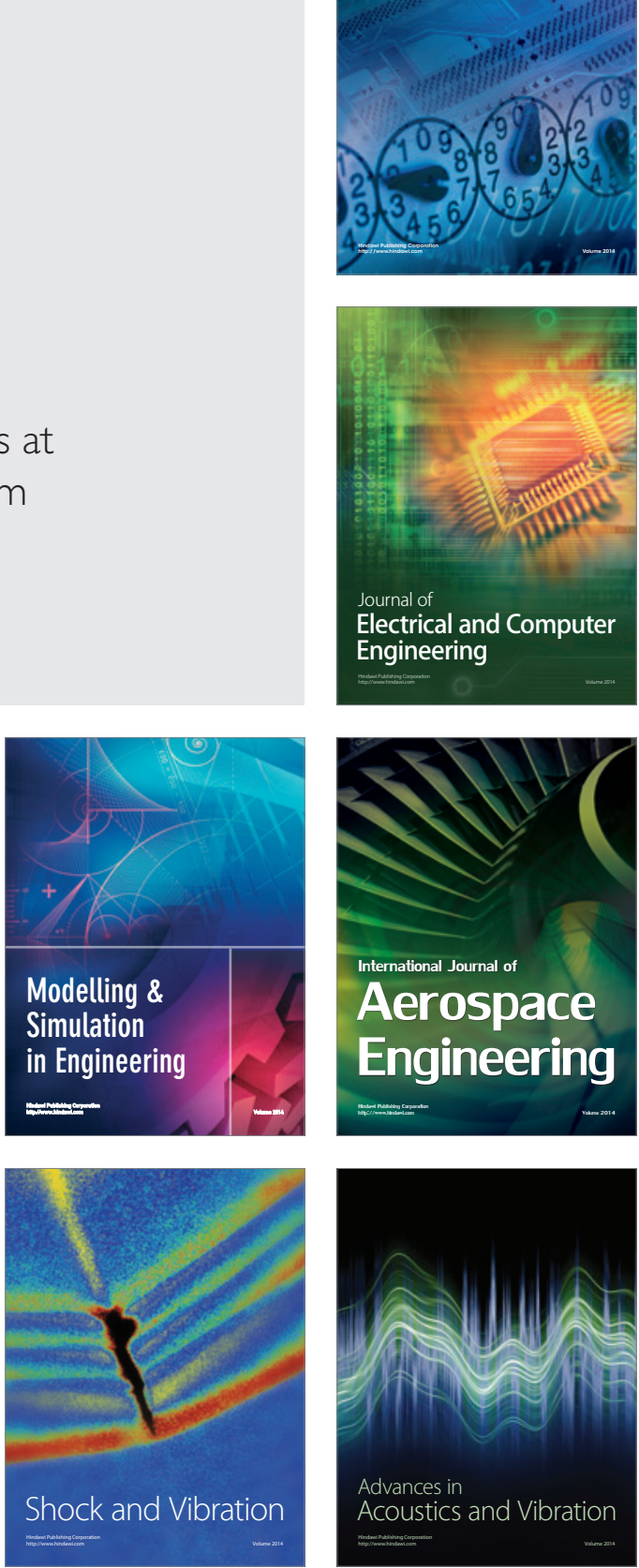